\title{
REACTION-DIFFUSION MODELS FOR BIOLOGICAL PATTERN FORMATION
}

\author{
E. J. CRAMPIN* AND P. K. MAINI*†
}

\begin{abstract}
We consider the use of reaction-diffusion equations to model biological pattern formation and describe the derivation of the reaction terms for several illustrative examples. After a brief discussion of the Turing instability in such systems we extend the model formulation to incorporate domain growth. Comparisons are drawn between solution behaviour on growing domains and recent results on self-replicating patterns on domains of fixed size.
\end{abstract}

1. Introduction. Understanding the mechanisms underlying spatial pattern formation is a central issue in early biological development. Although genes play a crucial role in embryology, a study of genetics alone may not be sufficient to reveal how the physical and chemical properties of embryonic material interact to produce the complex spatio-temporal signalling cues which ultimately determine cell fate. A useful tool for studying such complex nonlinear interactions is mathematical modelling, and there is now a vast literature on mathematical models of biological pattern formation.

In general, models for biological pattern formation describe either mechanisms of cell motility or the generation of chemical prepatterns. Cell movement models consider the aggregation of cell populations under chemical signalling cues or mechanical forces, with subsequent cell differentiation in response to increased cell density (for a review see [22]). The aggregation of cells may occur in response to a gradient in the distribution of a chemical signal (chemotaxis, see for example [18]) or may be due to mechanical cues: for example the advective forces exerted by motile cells on a substratum or via cellular motion up a gradient in adhesivity of the extracellular material (haptotaxis, see for example [27, 22]).

Chemical prepattern models, on the other hand, argue that a pattern is first established in the concentration of certain chemicals (morphogens), and subsequent cellular differentiation into different tissue types follows according to whether or not the local concentration exceeds some threshold. It is implicitly assumed in such models that the chemical pattern is established on a faster timescale than the response of the cellular machinery, so that the formation and interpretation of the pattern decouple.

Turing [39] demonstrated that such prepatterns could be formed in homogeneous media by a symmetry-breaking instability driven by diffusion. The model equations for two such interacting chemicals, $u(x, t)$ and $v(x, t)$, are the coupled reactiondiffusion equations

$$
\begin{aligned}
& \frac{\partial u}{\partial t}=D_{u} \nabla^{2} u+f(u, v) \\
& \frac{\partial v}{\partial t}=D_{v} \nabla^{2} v+g(u, v)
\end{aligned}
$$

where the kinetic functions $f$ and $g$ describe the nonlinear reaction between the chemicals. Turing showed this system can exhibit diffusion-driven instability whereby a spatially uniform steady state which is stable in the absence of diffusion can be driven UK.

*Centre for Mathematical Biology, Mathematical Institute, 24-29 St Giles', Oxford OX1 3LB,

†maini@maths.ox.ac.uk 
unstable by diffusion. Below we discuss several reaction kinetics that have been put forward for biological models. Then we consider recent developments in the reactiondiffusion theory for biological pattern formation where underlying domain growth has been suggested as an important mechanism in pattern selection, and relate these issues to recent results on self-replicating patterns in reaction-diffusion systems.

2. Biological and Biochemical Reaction Kinetics. Functional forms for the kinetic terms in models pertinent to biological pattern formation have been derived in several different ways. Below we describe several such derivations and provide illustrative examples.

2.1. Phenomenological Models. In their 1972 paper Gierer and Meinhardt [8] proposed several models for pattern formation in an attempt to explain the regenerative properties of hydra observed in various transplantation experiments. The model kinetics are based on biologically plausible arguments and functions are chosen to reproduce the experimentally observed behaviour. The scheme which has come to be known in the literature as the Gierer-Meinhardt model (described in their paper as activator-inhibitor model with different sources) considers autocatalytic activation of chemical $A(x, t)$ and self-inhibition of chemical $H(x, t)$ and has the form

$$
\begin{aligned}
& \frac{\partial A}{\partial t}=\rho_{0} \rho+c \rho \frac{A^{p}}{H^{q}}-\mu A+D_{A} \frac{\partial^{2} A}{\partial x^{2}} \\
& \frac{\partial H}{\partial t}=c^{\prime} \rho^{\prime} \frac{A^{r}}{H^{s}}-\nu H+D_{H} \frac{\partial^{2} H}{\partial x^{2}}
\end{aligned}
$$

where $0<(p-1) / q<r /(s+1)$. In their paper the authors consider all parameters to be positive constants except for the inhomogeneous distributed source terms $\rho(x)$ and $\rho^{\prime}(x)$, usually taken to be simple gradients across the solution domain. However, for constant parameters these kinetics may admit diffusion-driven instability. Standard values assumed for the powers in the quotients are $p=r=2, q=1$ and $s=0$, and the nondimensionalised kinetics may be written for $u=\nu c^{\prime} \rho^{\prime} H /(c \rho)^{2}$ and $v=c^{\prime} \rho^{\prime} A / c \rho$ as

$$
f(u, v)=v^{2}-u, \quad g(u, v)=\frac{v^{2}}{u}-k v+\delta
$$

where $u$ is the inhibitor (or substrate) and $v$ the activator, time is nondimensionalised with $\tau=\nu t, k=\mu / \nu$ and $\delta=c^{\prime} \rho_{0} \rho^{\prime} / c \nu$.

2.2. Hypothetical (Bio)chemical Reactions. Several authors have derived reaction schemes from descriptions of hypothetical chemical reactions. For example, Schnakenberg [34] proposed a series of trimolecular autocatalytic reactions involving two chemicals as follows

$$
X \underset{k_{2}}{\stackrel{k_{1}}{\rightleftharpoons}} A, \quad B \stackrel{k_{3}}{\longrightarrow} Y, \quad 2 X+Y \stackrel{k_{4}}{\longrightarrow} 3 X .
$$

Using the law of mass action and denoting the concentrations of $X, Y, A$ and $B$ by $x, y, \alpha$ and $\beta$, respectively, we have

$$
\frac{d x}{d t}=k_{2} \alpha-k_{1} x+k_{4} x^{2} y, \quad \frac{d y}{d t}=k_{3} \beta-k_{4} x^{2} y
$$

where $k_{1}, \ldots, k_{4}$ are (positive) rate constants. Assuming that there is an abundance of the external reactants $A$ and $B, \alpha$ and $\beta$ can be considered to be constant. After 
appropriate nondimensionalisation, with $u=\sqrt{k_{4}} y$ and $v=\sqrt{k_{4}} x$ representing the variation over time of the two concentrations respectively, then

$$
f(u, v)=b-u v^{2}, \quad g(u, v)=a+u v^{2}-v
$$

where $a=k_{2} \alpha / k_{1}$ and $b=k_{3} \sqrt{k_{4} / k_{1}} \beta / k_{1}$ are nondimensional parameters, and nondimensional time $\tau=k_{1} t$.

A further example used to study pattern formation is the Gray-Scott model for autocatalysis in the continuously fed unstirred flow reactor $[9,10,35]$ which may exhibit bistability. The kinetic scheme for this reaction is a variant of the autocatalytic model for glycolytic oscillations proposed by Sel'kov [37]. The scheme considers the autocatalytic production of $B$ which decays to form product $P$ in the irreversible reactions

$$
A+2 B \stackrel{k_{1}}{\longrightarrow} 3 B, \quad B \stackrel{k_{2}}{\longrightarrow} P .
$$

Here $B$ is self-activating (autocatalytic) while $A$ is a substrate for which higher concentrations increase the rate of its own removal. In a closed reactor, for which initial concentrations of $A$ and $B$ are specified and no material is allowed to enter or leave the reactor, eventually all of the reactants would be converted to product. However, nonequilibrium conditions may be maintained by a constant flow, at rate $\lambda$, through the reactor, with feed of reactant $A$ at a constant concentration $a_{0}$ and removal of the product $P$. The model equations take the form

$$
\frac{d a}{d t}=\lambda\left(a_{0}-a\right)-k_{1} a b^{2}, \quad \frac{d b}{d t}=-\lambda b+k_{1} a b^{2}-k_{2} b .
$$

After nondimensionalisation, under these nonequilibrium conditions, the kinetics are given for $u=a / a_{0}$ and $v=b / a_{0}$ by

$$
f(u, v)=F(1-u)-u v^{2}, \quad g(u, v)=-(F+k) v+u v^{2}
$$

where $k=k_{2} / k_{1} a_{0}^{2}$ is the effective rate constant for formation of product $P$ and $F=$ $\lambda / k_{1} a_{0}^{2}$ so that $\tau_{\text {res }}=k_{1} a_{0}^{2} t_{\text {res }}=1 / F$ is the mean residence time in nondimensional time units. By varying these two parameters the kinetics may have a single (trivial) steady state

$$
u_{r}=1, \quad v_{r}=0
$$

known as the red state. However, in contrast to the other models, this scheme may exhibit bistability when the discriminant $\Delta=1-4(F+k)^{2} / F>0$, giving two additional steady states arising in a saddle-node bifurcation

$$
\begin{aligned}
u_{b} & =\frac{1}{2}(1-\sqrt{\Delta}), & v_{b} & =\frac{F}{2(F+k)}(1+\sqrt{\Delta}) \\
u_{i} & =\frac{1}{2}(1+\sqrt{\Delta}), & v_{i} & =\frac{F}{2(F+k)}(1-\sqrt{\Delta})
\end{aligned}
$$

where the intermediate state $\left(u_{i}, v_{i}\right)$ is unstable and the blue state $\left(u_{b}, v_{b}\right)$ is stable.

This model has been widely studied, both as the simplest chemically plausible model which gives oscillations in the continuously stirred reactor and also in the context of chemical pattern formation in reaction-diffusion equations. It is similar in structure to the Brusselator model [23]. In the vicinity of the bistable regime the Gray-Scott model has been studied in the context of self-replicating phenomena, discussed below. 
2.3. Hypothetical models from Ecology. White and Gilligan [41] proposed a model for the population dynamics of a host-parasite-hyperparasite system to account for persistent spatio-temporal patterns in population densities in a homogeneous environment. The population dynamics is described by local interaction terms and diffusion is assumed to model the spatial spread and dispersion of each species. (Diffusion is commonly used as a model for the spatial spread of root systems and for the dispersal of spores.) In the field, patchiness has been observed for timescales much longer than those associated with stochastic heterogeneities (where eventually a uniform infestation of parasite would be expected). Phenomena monitored experimentally include drifting disease 'hot-spots' and periodic occurrence of disease at a particular location.

In dimensional form the local dynamics are governed for host $(H)$, parasite $(P)$ and hyperparasite $(Q)$ by the system

$$
\begin{aligned}
\frac{\mathrm{d} H}{\mathrm{~d} t} & =r H\left(1-\frac{H}{k}\right)-a P H \\
\frac{\mathrm{d} P}{\mathrm{~d} t} & =b P H-\frac{c P}{1+e P} Q \\
\frac{\mathrm{d} Q}{\mathrm{~d} t} & =l P-d Q
\end{aligned}
$$

where the host plant $H$ grows logistically and is removed by the parasite $P$ at a rate $a$ per unit parasite and has conversion factor $b$ per unit host. Predation of the hyperparasite $Q$ on the parasite is a saturating function of parasite population, with conversion at a rate $l$ per unit parasite, and the hyperparasite has a natural decay rate $d$. The parameters $r, k, c$ and $e$ are positive constants.

Following the authors we scale the population densities with their steady state values when $k=\infty$, namely $\left(Q_{s}^{\infty}, H_{s}^{\infty}, P_{s}^{\infty}\right)$, such that $u=H / H_{s}^{\infty}, v=P / P_{s}^{\infty}$ and $w=Q / Q_{s}^{\infty}$ and then the interaction kinetics are

$$
\begin{aligned}
f(u, v) & =u\left(1-\frac{u}{\kappa}\right)-u v \\
g(u, v, w) & =\mu\left(\frac{u v}{1+\beta}-\frac{v w}{1+\beta v}\right) \\
h(v, w) & =\delta(v-w)
\end{aligned}
$$

with the rescaled variables $\delta=d / r, \kappa=k / H_{s}, \mu=c Q_{s} / r$ and $\beta=b P_{s}$. Time is nondimensionalised with the rate parameter $r$. Under different parameter regimes this three-species model has been shown to demonstrate both stationary spatial patterns and patterns which oscillate in time.

2.4. Empirical Schemes. Here the kinetic term is obtained by modelling real data, where the function and model parameters are provided by fitting an experimental data curve. For example, the Thomas immobilized-enzyme substrate-inhibition mechanism $[38,1]$ involves the reaction of uric acid (concentration $u$ ) with oxygen (concentration $v$ ). Both reactants diffuse from a reservoir maintained at constant concentration $u_{0}$ and $v_{0}$, respectively, onto a membrane containing the immobilized enzyme uricase. They react in the presence of the enzyme with empirical rate $\frac{V_{m} u v}{\kappa_{m}+u+u^{2} / \kappa_{s}}$, where $V_{m}$ is a measure of the maximal reaction rate and $\kappa_{m}$ and $\kappa_{s}$ reflect the concentrations at which the enzyme is half activated and half inhibited 
respectively. The dynamics are then governed by

$$
\begin{aligned}
& \frac{d u}{d t}=p_{u}\left(u_{0}-u\right)-\frac{V_{m} u v}{\kappa_{m}+u+u^{2} / \kappa_{s}}, \\
& \frac{d v}{d t}=p_{v}\left(v_{0}-v\right)-\frac{V_{m} u v}{\kappa_{m}+u+u^{2} / \kappa_{s}}
\end{aligned}
$$

where $p_{u}$ and $p_{v}$ are transport coefficients for the reactants which are maintained by reservoir concentrations $u_{0}$ and $v_{0}$.

2.5. Models of Experimental Chemical Systems. Although Turing predicted the spatial patterning potential of chemical reactions in 1952, this phenomenon has only recently been realised in actual chemical reactions. It is now possible, in certain cases, to write down detailed reaction schemes.

The first Turing patterns were observed in the chlorite-iodide-malonic acid (CIMA) reaction with starch $[2,6]$. The model proposed by Lengyel and Epstein [17] stresses three processes: the reaction between malonic acid (MA) and iodine to create iodide, and the reactions between chlorite and iodide and chloride and iodide. These reactions take the form

$$
\begin{gathered}
\mathrm{MA}+\mathrm{I}_{2} \rightarrow \mathrm{IMA}+\mathrm{I}^{-}+\mathrm{H}^{+} \\
\mathrm{ClO}_{2}+\mathrm{I}^{-} \rightarrow \mathrm{ClO}_{2}^{-}+\frac{1}{2} \mathrm{I}_{2} \\
\mathrm{ClO}_{2}^{-}+4 \mathrm{I}^{-}+4 \mathrm{H}^{+} \rightarrow \mathrm{Cl}^{-}+2 \mathrm{I}_{2}+2 \mathrm{H}_{2} \mathrm{O}
\end{gathered}
$$

The rates of these reactions can be determined experimentally. By making the experimentally realistic assumption that the concentration of malonic acid, chlorine dioxide and iodine are constant, Lengyel and Epstein derived the following model

$$
\begin{aligned}
& \frac{\partial u}{\partial t}=k_{1}-u-\frac{4 u v}{1+u^{2}}+\nabla^{2} u \\
& \frac{\partial v}{\partial t}=k_{2}\left[k_{3}\left(u-\frac{u v}{1+u^{2}}\right)+c \nabla^{2} v\right]
\end{aligned}
$$

where $u, v$ are the concentrations of iodide and chlorite, respectively and $k_{1}, k_{2}, k_{3}$ and $c$ are positive constants.

3. Model Behaviour. We have described several illustrative examples, however, many more reaction schemes have been proposed (see, for example, [22]). Reaction-diffusion model behaviour has been extensively studied in the linearized regime on regular geometries. The response to small amplitude perturbations is determined by linearization about the uniform steady state, such that in this regime the solution consists of the amplitude growth-rate weighted sum of eigenfunctions of the Laplacian satisfying the boundary conditions, typically taken to be no-flux. To understand the behaviour of the full system, nonlinear perturbation analysis may be employed in the weakly nonlinear case, however, for the fully nonlinear case most investigations have required numerical simulation. In one spatial dimension, the patterns exhibited by these models are spatially periodic, with the number of oscillations increasing with domain length. On two-dimensional domains, stripes (analogous to the one-dimensional behaviour) are observed, as well as spots, the form of the nonlinearity being crucial in determining which type of behaviour is observed [7]. More 
complicated labyrinthine patterns may also be generated. It should also be noted that models can exhibit Turing-Hopf bifurcation, resulting in travelling peaks and troughs.

These models have been widely applied to spatial pattern formation in early development, including animal coat markings [21], limb development [19], shell patterns [20] and butterfly wing patterns [22, 36]. All of these applications consider pattern to arise in response to small amplitude spatial perturbations to the uniform steady state.

4. Reflection and Splitting of Travelling Pulses in the Gray-Scott Model. Turing's theory for morphogenesis considers the growth of infinitesimal perturbations driven by the diffusive instability. In general, however, reaction-diffusion systems can display a wider variety of spatio-temporal phenomena in response to large amplitude perturbations. The nature of the kinetic terms plays the major role in determining the solution behaviour.

The Gray-Scott model is an example of a kinetic scheme which, for suitably chosen parameters, demonstrates bistability. In the vicinity of the bistable regime, many interesting spatio-temporal phenomena have been reported. Pearson [29] first identified self-replication of spot-like patterns in two-dimensional numerical simulations of the Gray-Scott model, amongst various other behaviours, some exhibiting spatio-temporal chaos (typically from the interaction of Turing and Hopf modes). This behaviour was observed for sufficiently large perturbation away from the trivial (red) homogeneous steady state, and in a parameter regime in the vicinity of both a Hopf bifurcation in the kinetics, and a subcritical Turing bifurcation for the nontrivial steady state. Many of the patterns reported by Pearson had previously been or were subsequently identified in chemical experiments on the FIS reaction by Lee, Swinney and co-workers $[14,15]$; for a review see [16].

Corresponding phenomena in spatially one-dimensional systems have been studied numerically $[30,31,32]$. Depending on the precise location in parameter space (while remaining in the vicinity of the curve bounding the bistable region) these authors have reported various behaviours for finite initial perturbations. Travelling pulses which are reflected from zero flux boundaries are found in a region for which there is a unique steady state, reproduced in Figure 4.1(a). Also it is found that these pulses are reflected during collisions with other pulses [30]. Wave splitting (or self-replication of pulses), illustrated in Figure 4.1(b), is observed for small enough activator diffusion coefficient in the bistable region and, for parameters for which there is a single stable branch, in the vicinity of the saddle-node bifurcation. For the latter case Petrov et al [30] have suggested that in this region the kinetics show excitable dynamics, where small perturbations decay exponentially to the globally stable steady state but sufficiently large perturbations result in long refractory excursions through the phase space before returning to the fixed point. They suggest that by decreasing the activator diffusivity the wave back becomes decreasingly refractory, until finally it excites a secondary wave which moves off in the opposite direction. However, Nishiura and Ueyama [24] have shown that the splitting phenomena may be recovered for near equal activator and inhibitor diffusion coefficients, by judicious choice of the kinetic parameters.

Several authors have investigated analytically the pulse-splitting phenomenon in the Gray-Scott model. Osipov and Severtsev [25] discuss splitting and periodic patterns on infinite domains. Reynolds et al [33] present a heuristic explanation for self-replication of spots and analyse equations of motion for travelling pulses in the limit of small activator diffusion. Nishiura and Ueyama provide an argument based on 


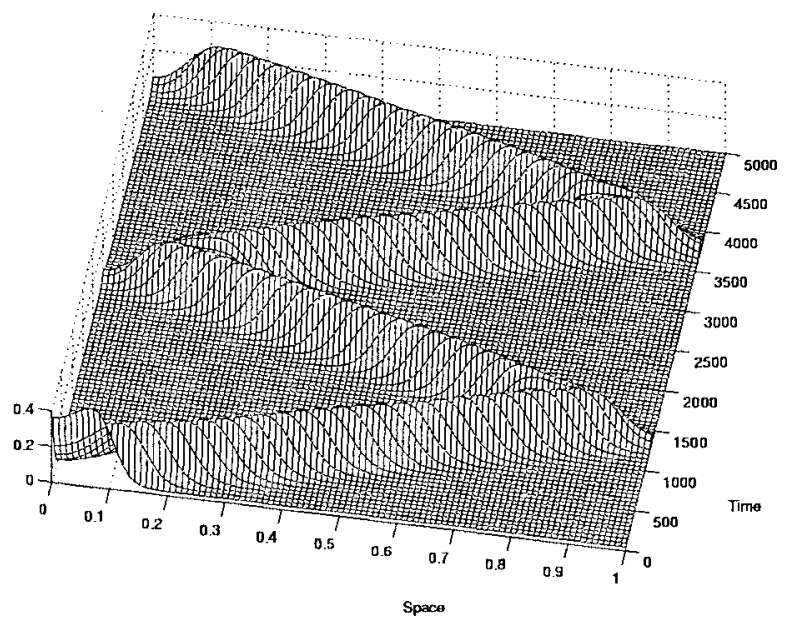

(a)

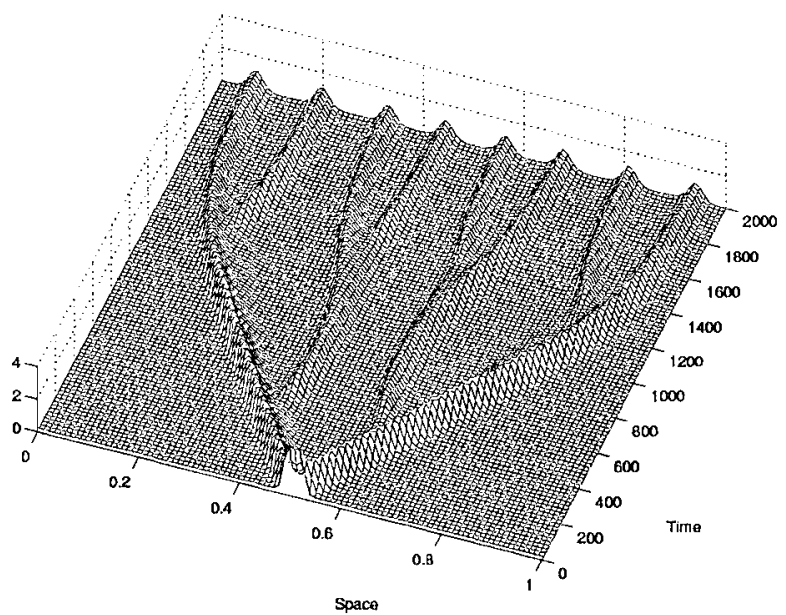

(b)

FIG. 4.1. Dynamic phenomena in the Gray-Scott model: (a) Travelling wave reflection at zero flux boundaries (parameters from [24]), with $F=0.025, k=0.0544$ (monostable region), $D_{u}=1.0$ and $D_{v}=0.5$ with domain length 112. (b) Wave-splitting (parameters from [32]) with $F=0.02$, $k=0.059$ (bistable region), $D_{u}=1.0$ and $D_{v}=0.01$ and domain length 60 .

underlying 'hidden' symmetries in the bifurcation structure of such reaction-diffusion equations. The authors show that the transient dynamics from the initial perturbation move in the vicinity of saddle-node bifurcations for steady Turing structures, which is reflected in the splitting process observed. Finally we note that although there is no supercritical Turing bifurcation for either solution branch, the transient splitting of travelling pulses may lead to steady Turing-type structures, which appear in a saddle-node bifurcation rather than through the diffusion-driven instability, 


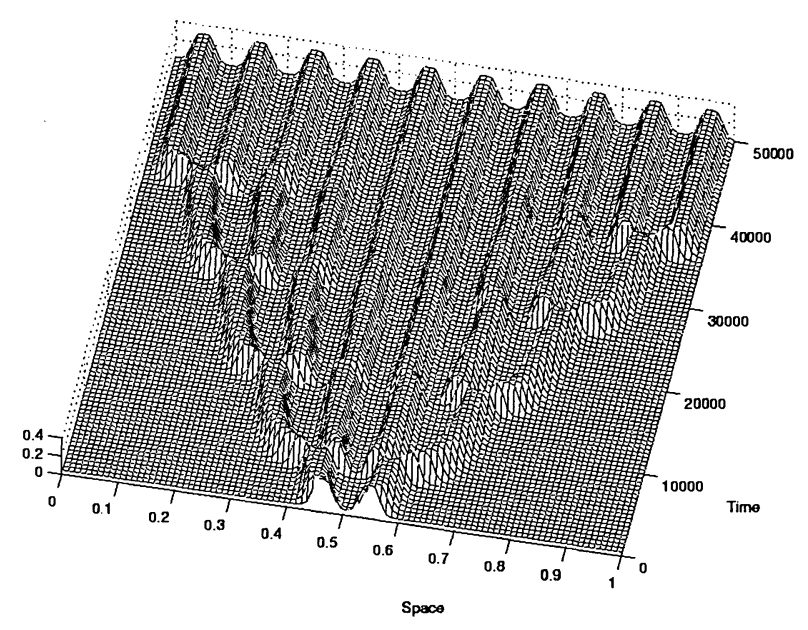

(a)

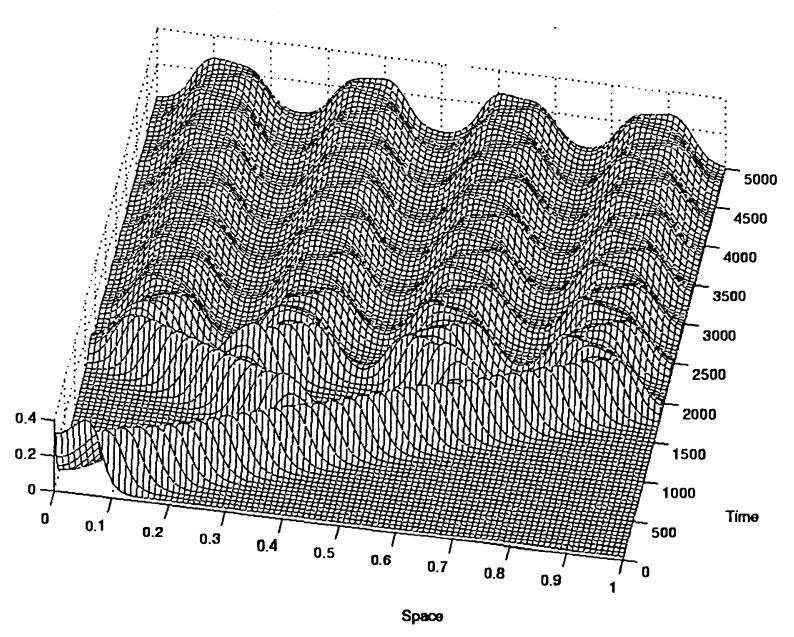

(b)

FIG. 4.2. Establishment of (a) steady and (b) oscillatory heterogeneous patterns for the GrayScott model with (a) $F=0.04, k=0.06075$ (parameters from [24]) and (b) $F=0.025, k=0.0542$, for both of which the kinetics have a unique steady state (but are close to the bistable region), $D_{u}=1.0$ and $D_{v}=0.5$. In (a) domain length is 450 and in (b) 160 .

as shown in Figure 4.2(a). However, long-time behaviour may also give oscillating 'mixed mode' behaviour, as in the example shown in Figure 4.2(b). In order to excite these spatio-temporal phenomena a sufficiently strong initial perturbation is required to escape exponential relaxation back to the trivial homogeneous state. Furthermore, these behaviours are restricted to narrow regions of parameter space (in the vicinity of the bistable region) for models such as the Gray-Scott system which have a highly complicated bifurcation structure.

Large initial perturbations for the reaction-diffusion system with simpler kinetic 


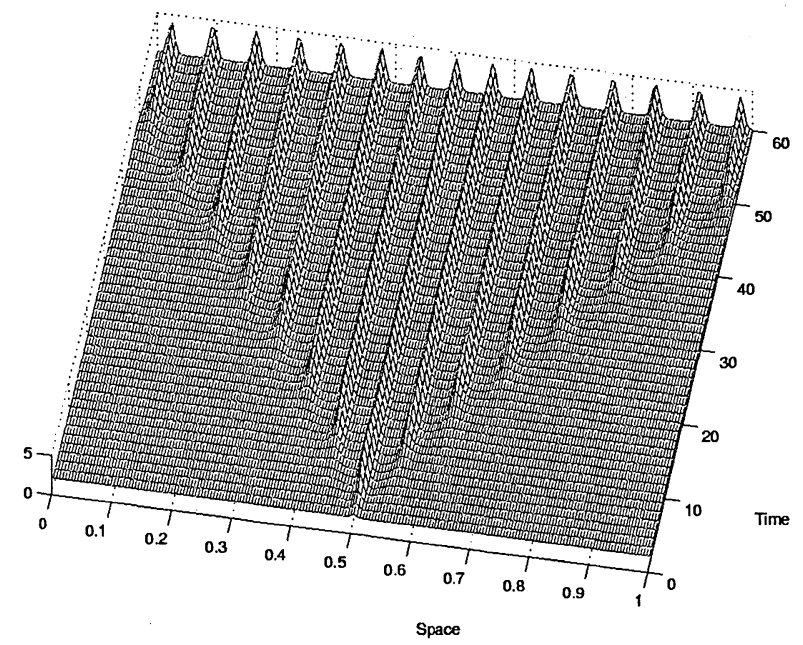

FIG. 4.3. Pattern selection behind a travelling front, initiated in the centre of the domain with homogeneous conditions elsewhere. For these simulations we use Schnakenberg kinetics with $a=0.1$, $b=0.9, D_{u}=1.0$ and $D_{v}=0.01$. The domain length is 40 .

schemes exhibiting a unique steady state do not, however, produce pulse splitting (demonstrated in Figure 4.3 for the Schnakenberg scheme). Rather, a periodic solution develops behind a travelling front. Similar related phenomena may, however, be generated for reaction-diffusion equations with Schnakenberg kinetics (and for other comparable systems) when pattern formation is driven by domain growth. Below we describe recent developments in reaction-diffusion theory on growing domains and illustrate the basic pattern formation phenomena.

5. Peak Splitting and Insertion in Reaction-Diffusion Patterns on Growing Domains. During development, pattern formation in the embryo is often accompanied by growth and movement of tissue, motivating consideration of domain growth in models of developmental patterning. Domain expansion has previously been considered in reaction-diffusion models for the branching morphology of growing unicellular algae [13] and for the emergence of tooth primordia in the alligator [12]. Here experimental evidence suggests that the spatial positioning and order of appearance of tooth primordia is determined in part by tissue growth throughout the jaw. Recently it has been suggested that reaction-diffusion prepatterns may be important in the growth and development of tumours [3].

New impetus was recently provided by Kondo and Asai [11], who suggested that a reaction-diffusion mechanism could be responsible for the dynamic changes in pigmentation patterns of the marine angelfish Pomacanthus. Unlike mammalian coat markings, the pattern in the skin of these fish changes dynamically during growth of the animal, rather than simply enlarging in proportion to the body size. Juvenile $P$. imperator display concentric stripes and $P$. semicirculatus have a regular array of vertical stripes which increase in number during growth. Juvenile $P$. semicirculatus of less than $2 \mathrm{~cm}$ in length display three vertical stripes which separate until the length of the fish is approximately $4 \mathrm{~cm}$, at which point new stripes appear between the original ones. Similarly at around $8-9 \mathrm{~cm}$ in length new stripes again appear between the existing ones. In this manner the pattern changes by insertion of new stripes as the animal roughly doubles in length, to preserve the wavelength of the pattern. In 
$P$. imperator this behaviour is maintained in the adult fish, where horizontal stripes maintain an average spacing.

A simple model of a growing tissue supposes that uniform tissue density is maintained, so that the expansion of the tissue may be described kinematically, with cell proliferation considered as a distributed source of volume. The equations for reaction and diffusion then become (see [4])

$$
\begin{aligned}
& \frac{\partial u}{\partial t}+\nabla \cdot(\underline{a} u)=D_{u} \nabla^{2} u+f(u, v) \\
& \frac{\partial v}{\partial t}+\nabla \cdot(\underline{a} v)=D_{v} \nabla^{2} v+g(u, v)
\end{aligned}
$$

where $\underline{a}(\underline{x}, t)$ is the velocity field generated by the distributed source term $S(\underline{x}, t)$ and

$$
\nabla \cdot \underline{a}=S(\underline{x}, t) .
$$

If all cells have the same rate of proliferation then $S(\underline{x}, t)=r$ is constant over the domain, and in one spatial dimension the domain length is given by $L(t)=\ell e^{r t}$. Transforming to a fixed interval, $z=x e^{-r t} \in[0, \ell]$ we recover, for $u(z, t)$ and $v(z, t)$,

$$
\begin{aligned}
& \frac{\partial u}{\partial t}=D_{u} e^{-2 r t} \frac{\partial^{2} u}{\partial z^{2}}+f(u, v)-r u \\
& \frac{\partial v}{\partial t}=D_{v} e^{-2 r t} \frac{\partial^{2} v}{\partial z^{2}}+g(u, v)-r v
\end{aligned}
$$

If the cell proliferation is not uniform across the domain then the advective term due to the domain growth will not disappear under this transformation. Biologically, it is reasonable to assume that domain growth is a much slower process than the reaction and diffusion of chemicals through the tissue. Therefore we assume that $r \ll 1$, the slow domain growth limit. Under these conditions two generic solution behaviours are observed, the regular splitting and insertion of new peaks in the activator solution, illustrated in Figure 5.1. Which of these two phenomena occurs will depend solely on the nonlinearities in the reaction kinetics.

The insertion of new peaks on the growing domain was compared by Kondo and Asai [11] to the regular insertion of stripes on the growing angelfish. Subsequent work $[40,28]$ has shown that stripe-forming equations on two-dimensional domains may exhibit similar behaviour. We have found that domain growth can stabilise parallel stripes, as well as giving rise to stripe splitting or insertion, and Painter et al [28] demonstrated that a population of cells responding chemotactically to the dynamic chemical prepattern can lead to the insertion of initially narrow stripes in the cell density, which broaden with age, as observed on the fish. While this is seemingly good circumstantial evidence that a mechanism such as reaction-diffusion is at work in the fish, it is important to note that these experimental observations may also be consistent with any other mechanism which generates patterns with an intrinsic wavelength.

6. Discussion. Pattern selection in the Turing instability on domains of fixed size displays strong sensitivity to the size and geometry of the solution domain. This has proved to be an important criticism of the application of Turing's ideas to some problems in biology where the number of wavelengths generated is critical. The root of this problem is in the fact that for domains of anything but very small aspect ratio (the ratio of domain size to intrinsic pattern wavelength) many patterns of different 


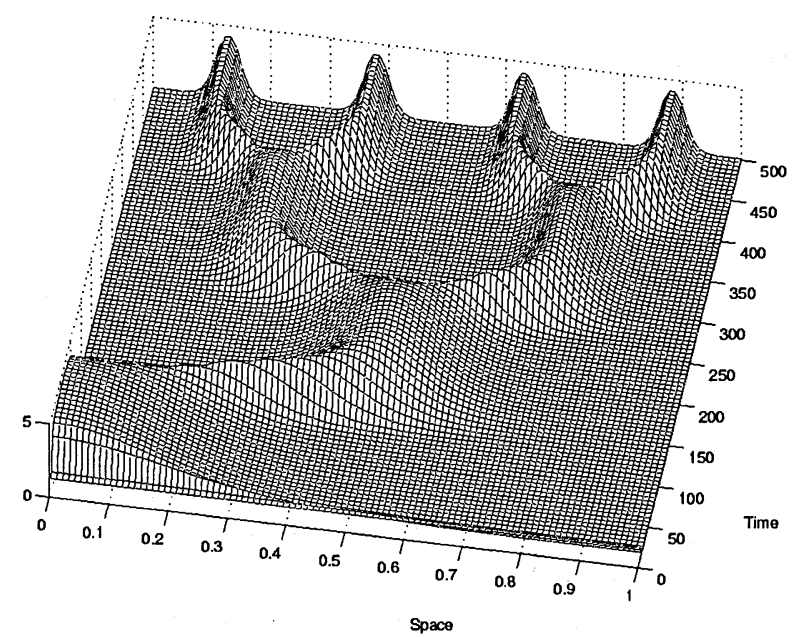

(a)

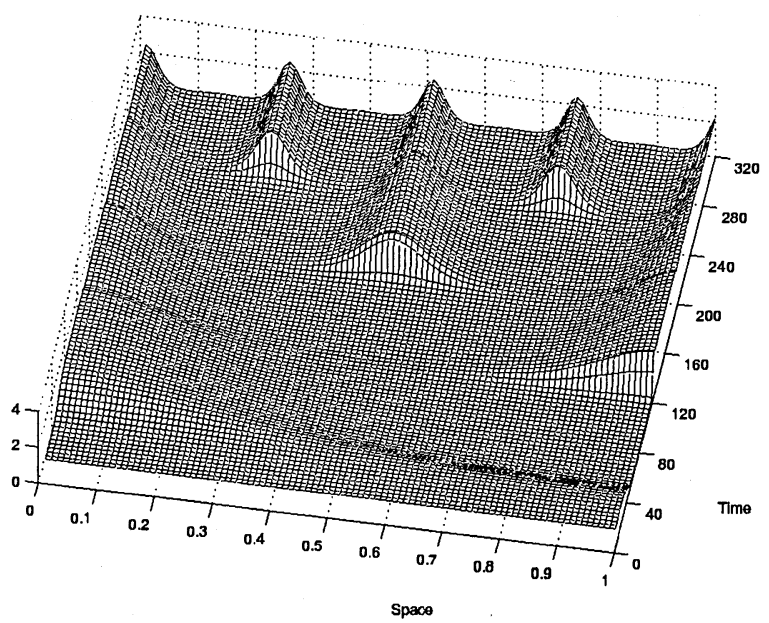

(b)

FIG. 5.1. Patterns in the activator chemical obtained on the expanding domain for (a) Schnakenberg kinetics (with $a=0.1, b=0.9$ ) showing peak splitting and (b) Gierer-Meinhardt kinetics $(k=0.5, \delta=0.05)$ showing regular insertion of new activator peaks. In both cases $D_{u}=1.0$, $D_{v}=0.01$ and $r=0.005$. Patterns change regularly through frequency (or mode) doubling behaviour.

wavenumber may be admitted as solutions in the dispersion relation, the number increasing as the dómain size is increased. Selection between these different patterns depends sensitively on initial data and domain geometry. In general, biological systems are subject to natural variation in parameters such as domain size, and reliable pattern generation requires a certain degree of scale invariance. We have suggested [4] 
that the regular doubling of the number of peaks on the domain can provide a mechanism for reliable pattern selection. Between the splitting or insertions, the pattern remains unchanged as the domain length changes, providing a degree of regulatory invariance to the domain size. Such results may prove useful when trying to identify which biological pattern formation events might arise as a consequence of the reaction-diffusion mechanism, and where it may be more prudent to seek alternative explanations.

Growing two-dimensional domains give rise to the division or insertion of spots or stripes, depending on the nonlinear kinetic terms. The arrangement of spot divisions, for example, is dependent on the geometry of the domain and the domain growth, where a single spot in the activator concentration profile may split into two, four or even six spots according to the symmetry of the domain. Such peak splitting and spot division behaviour is very reminiscent of the self-replication phenomena described above. The pattern formation process on the slowly growing domain moves between a quasi-steady regime, where the solution shadows the steady-state solution branch, and transitions between branches corresponding to splitting or insertion of activator peaks. These dynamics are driven by the increasing domain length, where the quasi-steady solution is taken to the point at which the steady solution branch disappears in a saddle-node bifurcation. The manner in which this critical point is reached depends on the nonlinearities in the kinetics [5]. The sequence of transitions between quasi-steady patterns can be understood using similar symmetry arguments [4] to those suggested by Nishiura and Ueyama [24] for self-replicating spots.

It is still a matter of controversy as to which is the correct theory for biological pattern formation, and it is almost certainly the case that different mechanisms are employed in different situations. From a mathematical viewpoint it has been shown that reaction-diffusion and cell motility (mechano-chemical) models exhibit very similar behaviour under linearization, suggesting that predictions can be made that are mechanism-independent [26]. In the nonlinear regime it is known that, for example, chemotaxis models exhibit blow-up and certain chemical prepattern models display spike solutions. However, it is still an open question as to whether solution behaviour in the nonlinear regime can be used to distinguish between model mechanisms. This raises the question as to whether the behaviour we have described on the growing domain is particular to reaction-diffusion systems or is generic to all global pattern generation mechanisms, such as chemotaxis and mechano-chemical models. If model predictions are found to differ for these other mechanisms then the solution behaviour on the growing domain will provide a useful means to distinguish between them. Whether these other models exhibit qualitatively different behaviour in response to domain growth is the subject of current investigation.

Acknowledgements. EJC acknowledges an earmarked studentship from the BBSRC.

\section{REFERENCES}

[1] B. Bunow, J-P. Kernevez, G. Joly, and D. Thomas, Pattern formation by reaction-diffusion instabilities: Applications to morphogenesis in Drosophila, J. theor. Biol., 84 (1980), pp. 629-649.

[2] V. Castets, E. Dulos, J. Boissonade, And P. De Kepper, Experimental evidence of a sustained Turing-type nonequilibrium chemical pattern, Phys. Rev. Lett., 64 (24) (1990), pp. 2953-2956. 
[3] M. A. J. Chaplain, M. Ganesh, And I. G. Graham, Spatio-temporal pattern formation on spherical surfaces: Numerical simulation and application to solid tumour growth, J. Math. Biol, 42 (5) (2001), pp. 387-423.

[4] E. J. Crampin, E. A. Gaffney, And P. K. Maini, Pattern formation through reaction and diffusion on growing domains: Scenarios for robust pattern formation, Bull. Math. Biol., 61 (1999), pp. 1093-1120.

[5] E. J. Crampin, E. A. Gaffney, And P. K. Maini, Mode doubling and tripling in reactiondiffusion patterns on growing domains: A piece-wise linear model, J. Math. Biol., to appear.

[6] P. De Kepper, V. Castets, E. Dulos, And J. Boissonade, Turing-type chemical patterns in the chlorite-iodide-malonic acid reaction, Physica D, 49 (1991), pp. 161-169.

[7] B. ERmentrout, Spots or stripes? Nonlinear effects in bifurcation of reaction-diffusion equations on the square, Proc. R. Soc. Lond. A, 434 (1991), pp. 413-417.

[8] A. Gierer and H. Meinhardt, A theory of biological pattern formation, Kybernetik, 12 (1972), pp. 30-39.

[9] P. GRAY AND S. K. SCOTT, Autocatalytic reactions in the isothermal, continuous stirred tank reactor: isolas and other forms of multistability, Chem. Eng. Sci, 38 (1) (1983), pp. 29-43.

[10] P. Gray AND S. K. ScotT, Autocatalytic reactions in the isothermal, continuous stirred tank reactor: Oscillations and instabilities in the system $A+2 B \rightarrow 3 B, B \rightarrow C$, Chem. Eng. Sci, 39 (6) (1984), pp. 1087-1097.

[11] S. KONDO AND R. AsAI, A reaction-diffusion wave on the skin of the marine angelfish Pomacanthus, Nature, 376 (1995), pp. 765-768.

[12] P. M. Kulesa, G. C. Cruywagen, S. R. Lubkin, P. K. Maini, J. Sneyd, M. W. J. Ferguson, AND J. D. MURRAY, On a model mechanism for the spatial pattering of teeth primordia in the alligator, J. theor. Biol., 180 (1996), pp. 287-296.

[13] T. C. LACALli, Dissipative structures and morphogenetic pattern in unicellular algae, Phil. Trans. R. Soc. Lond. B, 294 (1981), pp. 547-588.

[14] K. J. LeE, W. D. MCCormick, J. E. Pearson, And H. L. Swinney, Experimental observation of self-replicating spots in a reaction-diffusion system, Nature, 369 (6477) (1994), pp. 215218.

[15] K. J. LEE AND H. L. SwINNEY, Lamellar structures and self-replicating spots in a reactiondiffusion system, Phys. Rev. E, 51 (3A) (1995), pp. 1899-1915.

[16] K. J. LEE AND H. L. SwINNEY, Replicating spots in reaction-diffusion systems, Int. J. Bifurcation and Chaos, 7 (5) (1997), pp. 1149-1158.

[17] I. LENGYEL AND I. R. EPSTEIN, Modeling of Turing structures in the chlorite-iodide-malonic acid-starch reaction system, Science, 251 (1991), pp. 650-652.

[18] P. K. Maini, M. R. Myerscough, K. H. Winters, and J. D. Murray, Bifurcating spatially heterogeneous solutions in a chemotaxis model for biological pattern generation, Bull. Math. Biol., 53 (5) (1991), pp. 701-719.

[19] P. K. MAINI AND M. Solursh, Cellular mechanisms of pattern formation in the developing limb, Int. Rev. Cytol., 129 (1991), pp. 91-133.

[20] H. MEINhardT, The Algorithmic Beauty of Sea Shells, Springer, Heidelberg, 1995.

[21] J. D. MURRAY, A pre-pattern formation mechanism for animal coat markings, J. theor. Biol., 88 (1981), pp. 161-199.

[22] J. D. Murray, Mathematical Biology, Springer-Verlag, Berlin, 2nd edition, 1993.

[23] G. Nicolis AND I. Prigogine, Self-Organization in Nonequilibrium Systems, WileyInterscience, New York, 1977.

[24] Y. NishiURA AND D. UEYAMA, A skeleton structure of self-replicating dynamics, Physica D, 130 (1999), pp. 73-104.

[25] V. V. Osipov And A. V. Severtsev, Theory of self-replication and granulation of spike autosolitons, Phys. Lett. A, 222 (1996), pp. 400-404.

[26] G. F. Oster And J. D. Murray, Pattern formation models and developmental constraints, J. exp. Zool., 251 (1989), pp. 186-202.

[27] G. F. Oster, J. D. Murray, AND A. K. HARris, Mechanical aspects of mesenchymal morphogenesis, J. Embryol. Exp. Morph., 78 (1983), pp. 83-125.

[28] K. J. PAinter, P. K. MAini, ANd H. G. OThmer, Stripe formation in juvenile Pomacanthus explained by a generalised Turing mechanism with chemotaxis, Proc. Natl. Acad. Sci. USA, 96(1999), pp. 5549-5554.

[29] J. E. PeArson, Complex patterns in a simple system, Science, 261 (1993), pp. 189-192.

[30] V. Petrov, S. K. Scott, AND K. Showalter, Excitability, wave reflection, and wave splitting in a cubic autocatalysis reaction-diffusion system, Phil. Trans. R. Soc. Lond., A 347 (1994), pp. 631-642. 
[31] K. E. Rasmussen, W. Mazin, And E. Mosekilde, Wave-splitting in the bistable Gray-Scott model, Int. J. Bifurcation and Chaos, 6 (6) (1996), pp. 1077-1092.

[32] W. N. Reynolds, J. E. PeARson, And S. Ponce-DaWson, Dynamics of self-replicating patterns in reaction diffusion systems, Phys. Rev. Lett., 72 (17) (1994), pp. 2797-2800.

[33] W. N. Reynolds, S. Ponce-Dawson, and J. E. Pearson, Self-replicating spots in reactiondiffusion systems, Phys. Rev. E, 56 (1) (1997), pp. 185-198.

[34] J. Schnakenberg, Simple chemical reaction systems with limit cycle behaviour, J. theor. Biol., 81 (1979), pp. 389-400.

[35] S. K. Scott, Chemical Chaos, Clarendon Press, Oxford, 1991.

[36] T. Sekimura, A. Madzvamuse, A. J. Wathen, and P. K. Maini, A model for colour pattern formation in the butterfly wing of Papilio dardanus, Proc. R. Soc. Lond., B 267 (2000), pp. 851-859.

[37] E. E. SEL'Kov, Self-oscillations in glycolysis: 1. A simple kinetic model, Eur. J. Biochem., 4 (1986), pp. 79-86.

[38] D. ThomAs, Artificial enzyme membranes, transport, memory and oscillatory phenomena, In D. Thomas and J.-P. Kernevez, editors, Analysis and Control of Immobilised Enzyme Systems, Springer, Berlin, 1976, pp. 115-150.

[39] A. M. Turing, The chemical basis of morphogenesis, Phil. Trans. R. Soc. Lond. B, 237 (1952), pp. 37-72.

[40] C. Varea, J. L. Aragón, and R. A. Barrio, Confined Turing patterns in growing systems, Phys. Rev. E, 56 (1) (1997), pp. 1250-1253.

[41] K. A. J. White And C. A. Gilligan, Spatial heterogeneity in three-species, plant-parasitehyperparasite, systems, Phil. Trans. R. Soc. Lond. B, 353 (1998), pp. 543-557. 